\title{
Diffusion Tensor Imaging and Fiber Tractography in Children with Craniosynostosis Syndromes
}

\author{
B.F.M. Rijken, A. Leemans, Y. Lucas, K. van Montfort, I.M.J. Mathijssen, and M.H. Lequin
}

\begin{abstract}
BACKGROUND AND PURPOSE: Patients with craniosynostosis syndromes caused by mutations in FGFR-2, FGFR-3, and TWIST7 genes are characterized by having prematurely fused skull sutures and skull base synchondroses, which result in a skull deformity and are accompanied by brain anomalies, including altered white matter microarchitecture. In this study, the reliability and reproducibility of DTI fiber tractography was investigated in these patients. The outcomes were compared with those of controls.
\end{abstract}

MATERIALS AND METHODS: DTI datasets were acquired with a 1.5T MR imaging system with 25 diffusion gradient orientations (voxel size $=1.8 \times 1.8 \times 3.0 \mathrm{~mm}^{3}$, b-value $=1000 \mathrm{~s} / \mathrm{mm}^{2}$ ). White matter tracts studied included the following: corpus callosum, cingulate gyrus, fornix, corticospinal tracts, and medial cerebellar peduncle. Tract pathways were reconstructed with ExploreDTI in 58 surgically treated patients with craniosynostosis syndromes and 7 controls (age range, 6-18 years).

RESULTS: Because of the brain deformity and abnormal ventricular shape and size, DTI fiber tractography was challenging to perform in patients with craniosynostosis syndromes. To provide reliable tracts, we adapted standard tracking protocols. Fractional anisotropy was equal to that in controls ( 0.44 versus $0.45 \pm 0.02, P=.536$ ), whereas mean, axial, and radial diffusivity parameters of the mean white matter were increased in patients with craniosynostosis syndromes $(P<.001)$. No craniosynostosis syndrome-specific difference in DTI properties was seen for any of the fiber tracts studied in this work.

CONCLUSIONS: Performing DTI fiber tractography in patients with craniosynostosis syndromes was difficult due to partial volume effects caused by an anisotropic voxel size and deformed brain structures. Although these patients have a normal fiber organization, increased diffusivity parameters suggest abnormal microstructural tissue properties of the investigated white matter tracts.

ABBREVIATIONS: $A D=$ axial diffusivity; $F A=$ fractional anisotropy; $F T=$ fiber tractography; $M D=$ mean diffusivity; $R D=$ radial diffusivity

C

raniosynostosis occurs in 1:2100-2500 neonates, of which at least $20 \%$ is caused by an identified genetic mutation. FGFR-2 (32\%), FGFR-3 (25\%), and TWIST1 (19\%) are the most commonly involved genes, responsible for Apert and CrouzonPfeiffer, Muenke, and Saethre-Chotzen syndromes, respectively. Patients in whom $\geq 2$ cranial sutures have fused prematurely but

Received November 12, 2014; accepted after revision January 2, 2015.

From the Departments of Plastic and Reconstructive Surgery and Hand Surgery (B.F.M.R., Y.L., I.M.J.M.) and Radiology (M.H.L.), Erasmus Medical Center/Sophia Children's Hospital, Rotterdam, the Netherlands; Image Sciences Institute (A.L.), University Medical Center, Utrecht, the Netherlands; and Department of Biostatics (K.v.M.), Erasmus Medical Center, Rotterdam, the Netherlands.

The research of A.L. was supported by VIDI Grant 639.072.411 from the Netherlands Organisation for Scientific Research.

Please address correspondence to B.F.M. Rijken, MD, Erasmus MC Rotterdam, Department of Plastic and Reconstructive Surgery and Hand Surgery, Room Ee 15.91b, PO Box 2040, 3000 CA Rotterdam, the Netherlands; e-mail: b.rijken@erasmusmc.nl

- Indicates open access to non-subscribers at www.ajnr.org

= Indicates article with supplemental on-line tables.

http://dx.doi.org/10.3174/ajnr.A4301 for whom no responsible gene mutation has been found are referred to as having complex craniosynostosis $(5.5 \%) .{ }^{1}$

Patients with complex and syndromic craniosynostosis syndromes are characterized by the premature fusion of skull sutures and skull base synchondroses, which induces an abnormal growth of the skull, skull base, and midface. Not only are bony structures involved in craniosynostosis, but brain and CSF circulation appear to be directly affected by the genetic defect as well. ${ }^{2-5}$ Because genes responsible for craniosynostosis syndromes are expressed during early embryonic development of the head, ${ }^{6}$ it is likely that these intrinsic factors can also induce disturbances in microstructural WM organization. ${ }^{4,7}$

Structural or mechanical cerebral abnormalities such as Chiari malformation type I are often reported in these patients. ${ }^{8}$ Additionally, ventriculomegaly, hypoplasia of the corpus callosum or hippocampus, agenesis of the septum pellucidum, and even aberrations in WM are seen. ${ }^{4,9-13}$ Patients with craniosynostosis syndromes have a 2-fold higher risk for de- 
veloping intellectual disability than the normative population, while they also have more behavioral and emotional functioning problems. ${ }^{14}$

In this study, we investigated whether diffusion tensor imaging and fiber tractography (FT) can be used for studying WM organization in patients with craniosynostosis syndromes. This technique should give more objective and anatomically complete information about WM tracts than the subjective single ROI approach that has been used before, ${ }^{7}$ because parameters will be defined over the total length of a particular WM tract rather than at 1 certain location in the tract. Additionally, we wanted to focus on different types of fiber tracts (commissural, projection, and association) and to study whether DTI properties differ between patients with craniosynostosis syndromes and control subjects. Fractional anisotropy (FA), mean diffusivity (MD), axial diffusivity (AD), and radial diffusivity (RD) are common diffusion properties for characterizing fiber structural features and providing information about axonal tissue organization. ${ }^{15-17}$

We hypothesized that FA would be reduced and diffusivity properties would be increased in patients with craniosynostosis syndromes compared with controls; these changes would indicate an abnormal microstructural tissue organization.

\section{MATERIALS AND METHODS}

The medical ethics committee approved this prospective study (MEC-2014-461), performed at the Dutch Craniofacial Center, the national referral center for patients with craniosynostosis syndromes in a population of 16 million inhabitants. MR imaging data were acquired between July 2006 and October 2013 as part of the standard clinical follow-up protocol for patients with syndromic or complex craniosynostosis.

\section{Subjects}

In this study, we included 58 patients with craniosynostosis syndromes, including Apert, Crouzon-Pfeiffer, Muenke, and Saethre-Chotzen syndromes and patients with complex craniosynostosis. The latter group included patients who had at least 2 prematurely closed skull sutures, for which a responsible gene mutation has not yet been found. The study population incorporated that of Florisson et al. ${ }^{7}$ However, the inclusion period of this study was longer; therefore, we included more patients. In this study, we added 6 patients with Apert syndrome, 2 with CrouzonPfeiffer syndrome, 2 with Muenke syndrome, and 5 with complex craniosynostosis. Sixty-nine patients underwent both genetic testing and MR imaging, including DTI. Patients with craniosynostosis syndromes are at risk for developing episodes of increased intracranial pressure; therefore, they routinely underwent a cranial vault expansion within the first year of life to prevent or treat increased intracranial pressure. However, they still might develop enlarged ventricles, mostly because of disturbed CSF absorption due to venous hypertension. ${ }^{2}$ Enlarged ventricles can induce periventricular WM atrophy but also affect the shape of surrounding brain structures. Therefore, we included the frontal occipital horn ratio in our analyses to correct for ventricular size.

Only the first MR image of our patients with craniosynostosis syndromes between 6 and 18 years of age was included. In addi- tion, we included 7 healthy control subjects within the same age range who were previously neuropsychologically tested and scanned (identical scanner and MR imaging protocol) for another study. ${ }^{18}$ Exclusion criteria for all subjects were the following: insufficient quality of the collected DTI dataset due to incomplete scanning, motion artifacts, or inhomogeneity of the MR imaging field due to braces or metallic remains from operations. In total, we excluded 7 patients with craniosynostosis syndromes: 1 had Apert syndrome, 1 had Crouzon-Pfeiffer syndrome, 2 had Muenke syndrome, 1 had Saethre-Chotzen syndrome, and 2 had complex craniosynostosis. In addition, 1 patient with Apert and 3 with Crouzon-Pfeiffer syndrome with a ventriculoperitonealshunt were also excluded from the study because this may influence the specificity of the collected DTI dataset. Consequently, the final study population included 58 patients with craniosynostosis syndromes and 7 control subjects.

\section{Image Acquisition}

All MR imaging data were acquired with a $1.5 \mathrm{~T}$ unit (General Electric Healthcare, Milwaukee, Wisconsin), including 3D T1 spoiled gradient-recalled, 3D T2 Cube, and DTI sequences. DTI was obtained by using a multirepetition single-shot echo-planar sequence with a section thickness of $3 \mathrm{~mm}$ without a gap. Images were obtained in 25 gradient directions with the following parameters: sensitivity, $b=1000 \mathrm{~s} / \mathrm{mm}^{2}$; TR $=15,000 \mathrm{~ms}$; TE $=82.1 \mathrm{~ms}$; FOV $=240 \times 240 \mathrm{~mm}^{2}$; and matrix $=128 \times 128$, resulting in a voxel size of $1.8 \times 1.8 \times 3.0 \mathrm{~mm} .^{7}$ This protocol was identical throughout the entire study period.

\section{Data Collection}

All DTI processing was performed by using ExploreDTI (http:// exploredti.com/). ${ }^{19}$ In summary, processing consisted of correction of subject motion and eddy current distortions ${ }^{20}$ and a weighted linear least-squares estimation of the diffusion tensor with the robust extraction of kurtosis indices with linear estimation (REKINDLE) approach. ${ }^{21,22}$ The MRI Atlas of Human White Matter of Oishi et $\mathrm{al}^{23}$ was used as a guideline to reconstruct the fiber pathways. WM tracts for tractography included projection fibers (corticospinal tract), commissural/callosal fibers (corpus callosum, anterior commissure), association fibers (uncinate fasciculus), tracts of the limbic system (fornix, cingulate gyrus), and tracts in the brain stem (medial cerebellar peduncle).

Tractography was performed by placing ROIs on each dataset by using "OR/SEED" and "AND" operators to allow tracts to pass through, and "NOT" operators when tracts were not allowed to pass through. ${ }^{24}$ In addition, "NOT" operators were occasionally placed in the midline to avoid crossing fibers from other bundles. Furthermore, 2 ANDs were placed to extract a certain segment of a WM tract; thereby, identical tract parts of each subject were measured. The FA threshold was set at 0.1 , and the maximum angle threshold, at $45^{\circ}$. For all included WM tracts, ROI definitions were adjusted to established publications presenting good validity and reliability. ${ }^{25,26}$ The adapted protocol for DTI FT was exactly the same in both control and craniosynostosis groups and is described in more detail below. 

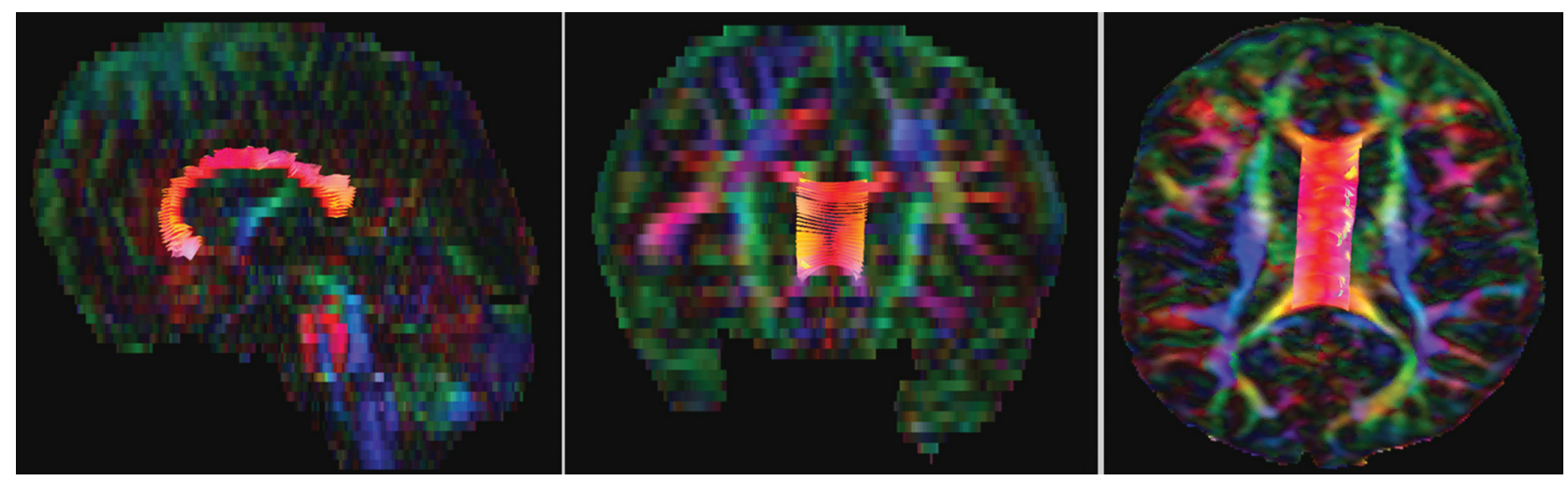

FIG 1. Midsagittal $10 \mathrm{~mm}$ of the corpus callosum in a 6-year-old female patient with Muenke syndrome.

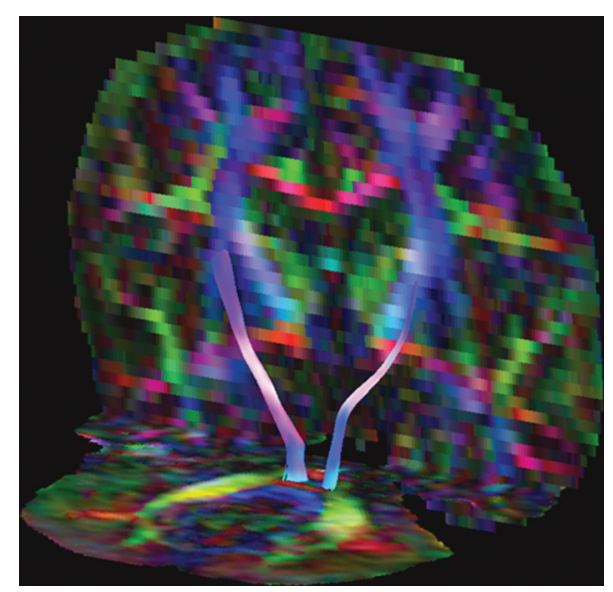

FIG 2. Midsegment of bilateral corticospinal tracts in a 6-year-old female patient with Muenke syndrome.

\section{Commissural Fibers}

Corpus Callosum. The genu, corpus, and splenium of the corpus callosum were measured separately; for all 3 parts, a OR/SEED was placed similarly in the midsagittal plane around the relevant part of the corpus callosum. To exclude regions of crossing fibers and partial volume effects, we set the maximum fiber length at 10 $\mathrm{mm}$; thus, only the midsagittal segment of the corpus callosum was selected (Fig 1). ${ }^{27}$

\section{Projection Fibers}

Corticospinal Tracts. Fiber tracts of the corticospinal tracts (Fig 2) were generated for both sides, by placing a OR/SEED at the level of the medial cerebellar peduncle, where it was clearly separated from the pontine crossing tract and corticospinal tracts. An AND was placed at the same level, 1 additional AND at the level of the decussation of the superior cerebellar peduncle, and 1 AND around the posterior limb of the internal capsule.

\section{Limbic System Fibers}

Cingulate Gyrus. The cingulate gyrus was divided into central and hippocampal parts (Fig $3 A,-B$ ). For the first part, a OR/SEED was placed in the coronal plane above the middle part of the corpus of the corpus callosum. Additionally, 1 AND was placed posterior to it, while another AND was placed anterior to the splenium; therefore, only the middle part of the cingulate gyrus was tracked. The hippocampal part was measured by a OR/SEED placed in the coronal plane underneath the splenium, followed by 1 AND placed in the transverse plane just below the splenium and a second AND, over the cingulate gyrus at the temporal lobe, which was already labeled by tracking.

Fornix. With regard to FT of the fornix, a OR/SEED was placed in the transverse plane around the turquois body of the fornix located at the level of the thalamus. An AND was placed at the body of the fornix in the coronal plane and separately in the transverse plane around the left or right bundle that was labeled by tracking. By placing 2 additional ANDs, 1 around the fornix at the level of the anterior commissure and the other at the level of the temporal lobe, an identical segment of the fornix was labeled in all patients (Fig 3C).

\section{Tracts in the Brain Stem}

Medial Cerebellar Peduncle. Fiber tracts of the medial cerebellar peduncle (Fig 4) were tracked by placing a OR/SEED around the relevant structure in the coronal view and by placing 2 ANDs around each peduncle at the level of the pontine crossing tract and posterior from the pons.

\section{Statistical Analysis}

A linear regression analysis was performed for each dependent variable: $\mathrm{FA}, \mathrm{MD}, \mathrm{AD}$, and $\mathrm{RD}$ of each $\mathrm{WM}$ structure, while tract volume and the frontal occipital horn ratio were added to the model as independent variables to correct for tract volume and ventricular size. Similar to findings in the study of Florisson et al, ${ }^{7}$ age did not have a statistically significant effect on DTI parameters and was therefore excluded from our model. In total, we tested 4 diffusion properties (FA, MD, $\mathrm{AD}$, and $\mathrm{RD}$ ) and $11 \mathrm{WM}$ structures (corpus callosum [genu, corpus, splenium, and total], bilateral cingulate gyrus, fornix, bilateral corticospinal tracts, medial cerebellar peduncle, and mean WM), resulting in 44 comparisons between patients with craniosynostosis syndromes and controls. A Bonferroni correction was performed, and a $P$ value $<.001(P$ value $=.05 / 44)$ was considered statistically significant. The intra- and interobserver reliability was tested by average-measures 2 -way mixed intraclass correlation coefficients. 

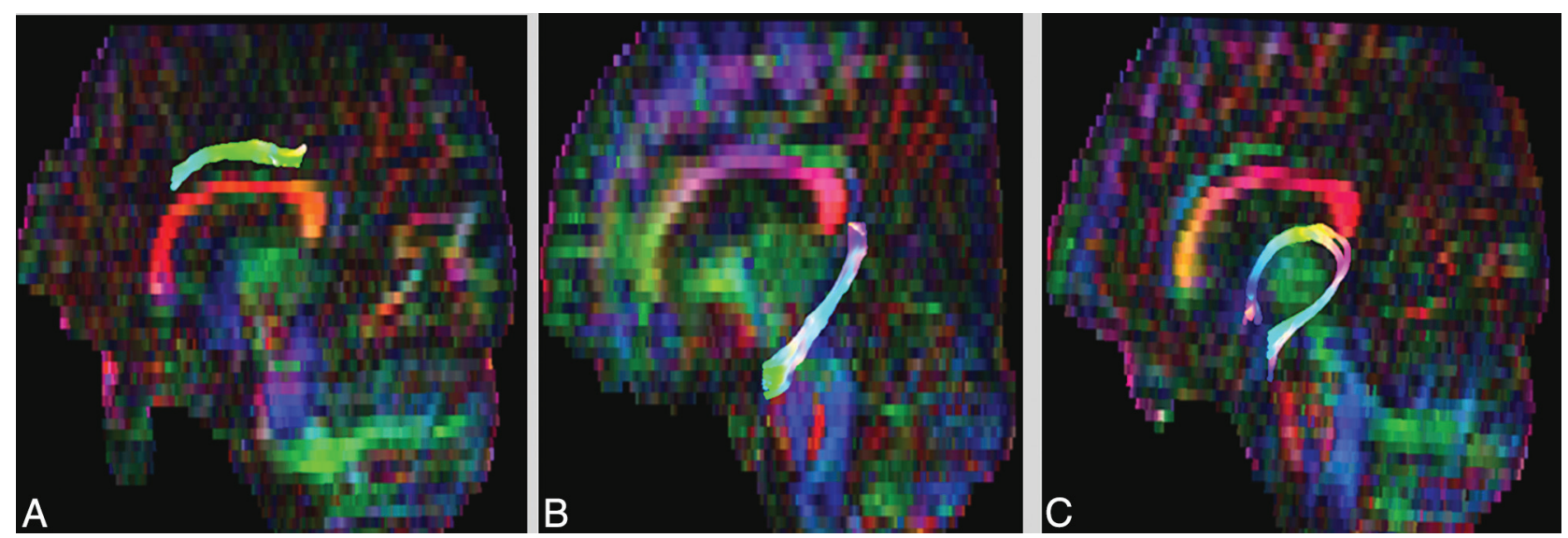

FIG 3. A, Central segment of cingulate gyrus. $B$, hippocampal segment of cingulate gyrus. $C$, Segmental part of the fornix.

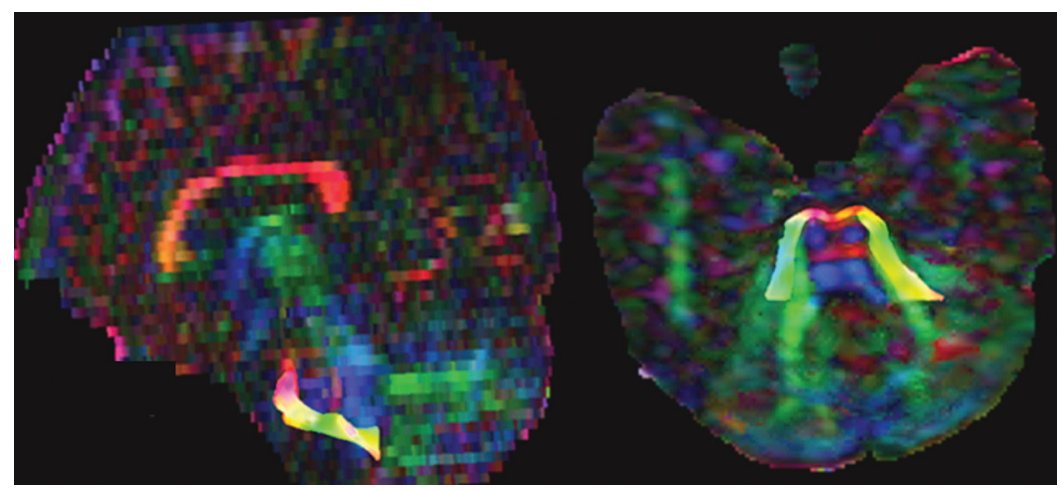

FIG 4. Segmental part of the medial cerebellar peduncle.

\section{Patient characteristics}

\begin{tabular}{lccccccc}
\hline \multicolumn{1}{c}{ Syndrome } & Apert & $\begin{array}{c}\text { Crouzon- } \\
\text { Pfeiffer }\end{array}$ & $\begin{array}{c}\text { Saethre- } \\
\text { Muenke }\end{array}$ & $\begin{array}{c}\text { Total } \\
\text { Chotzen }\end{array}$ & Complex & Craniosynostosis & Controls \\
\hline No. of subjects & 10 & 16 & 10 & 9 & 13 & 58 & 7 \\
M/F sex & $5: 5$ & $9: 7$ & $4: 6$ & $6: 3$ & $7: 6$ & $31: 27$ & $2: 5$ \\
Mean age (yr) & 11.1 & 10.1 & 7.9 & 8.9 & 8.9 & 9.4 & 10.7 \\
\hline
\end{tabular}

using 2 AND operators), we could measure identical WM structures and make fair comparisons between patients with craniosynostosis syndromes and control subjects. Unfortunately, the anterior commissure was not measurable in either controls or patients with craniosynostosis syndromes. Probably the anisotropic voxel size used in our protocol was too large to reconstruct such a small structure. The uncinate fasciculus showed implausible tractography in patients with craniosynostosis syndromes; therefore, both structures were excluded from the study. Furthermore, in different patients with craniosynostosis syndromes, fiber tracts could not be measured due to partial volume effects, mainly involving the cingulate gyrus and fornix (13 patients with Apert syndrome and 1 with Crouzon-Pfeiffer syndrome).

\section{RESULTS}

\section{Subjects}

This study included 7 control subjects (mean age, 10.7 years; range, $7.5-15$ years) and 58 patients with syndromic or complex craniosynostosis (mean age, 9.4 years; range, 6-18 years). The distribution for the different syndromes is presented in Table.

\section{Measurement: Reliability and Reproducibility}

DTI FT in patients with craniosynostosis syndromes was challenging because of partial volume effects due to the brain deformity and abnormal ventricular size and shape. Therefore, standard FT protocols could not be used, and measurements of all tracts needed to be adapted to track reliable and comparable fiber tracts in all subjects. Although an FA threshold of 0.2 is commonly used, an FA threshold of 0.1 made it possible to track all structures in the control group and almost all included structures in the craniosynostosis group. Consequently, by using an FA threshold of 0.1 , more aberrant tracts were generated and additional AND and NOT ROIs were required to exclude aberrant fibers. Additionally, by extracting particular segments from a WM tract (by

\section{Intra- and Interobserver Reliability}

Observers (B.F.M.R. and Y.L.) had 2 years of experience in DTI-FT and were supervised by A.L. with 12 years of experience in DTI and by M.H.L. with 20 years of experience in pediatric neuroradiology. Intraobserver reliability of measurements was determined by observer 1 (Y.L.), who performed all structural measurements twice in 10 subjects, 5 patients and 5 control subjects. This process resulted in an intraclass correlation coefficient of 0.93 . Interobserver reliability was measured by comparing the results of observer 1 with those of a second observer (B.F.M.R.), who measured the same 10 subjects; this procedure resulted in an interclass correlation coefficient of 0.94 .

\section{WM Tracts and Ventriculomegaly}

The frontal occipital horn ratio was more constant in control subjects (range, 0.31-0.37; mean, $0.35 \pm 0.02$ ) than in patients with craniosynostosis syndromes (range, 0.25-0.53; mean, $0.38 \pm 0.05)$ and was highest in patients with Apert and CrouzonPfeiffer syndromes, indicating that these patients had the largest 
ventricles. The frontal occipital horn ratio was not significantly correlated to the FA, MD, AD, and RD of mean WM. However, it was significantly correlated to a reduced FA and increased diffusivity properties of the genu and corpus of the corpus callosum $(P<.001)$, but it had no significant effect on the splenium.

\section{WM Measures and Craniosynostosis}

Mean WM DTI properties were calculated by the sum of the properties of the individual structures and divided by the number of brain structures that could be measured.

FA. The mean FA of the total group of patients with craniosynostosis syndromes showed an FA of the mean WM similar to that of control subjects ( 0.44 versus $0.45, P=.536)$, as well as for the separate WM tracts.

Diffusivity Properties. $\mathrm{MD}, \mathrm{AD}$, and $\mathrm{RD}$ of the mean WM were significantly higher in patients with craniosynostosis syndromes compared with the control group $(P<.001)$. Whereas all diffusivity properties were significantly increased in the cingulate gyrus and corticospinal tracts, only $\mathrm{AD}$ was significantly increased in the corpus and splenium of the corpus callosum (On-line Table 1).

With regard to the different craniosynostosis syndromes, FA of mean WM and separate WM tracts was equal to that of control subjects (On-line Table 2). However, $\mathrm{MD}, \mathrm{AD}$, and RD of mean WM were significantly higher in each syndrome compared with controls $(P<.001)$. While the cingulate gyrus and corticospinal tracts were affected the most, diffusivity properties of the fornix and medial cerebellar peduncle were similar between each craniosynostosis group and the control group. For an overview see Online Tables 3-5.

\section{DISCUSSION}

Following DTI studies on ROIs, we now performed DTI FT to study WM tracts in patients with syndromic and complex craniosynostosis. The aim of the study was to investigate the reliability of this technique in an unusual patient group with skull and brain deformities and to compare DTI properties between these patients and controls. Due to the premature fusion of skull sutures, patients with craniosynostosis syndromes develop abnormal skull and brain shapes. For instance, when coronal sutures are involved, a brachycephalic skull shape will develop. Consequently, brain structures running in the anteroposterior direction will be bent or even compressed, while structures running from left to right might be stretched out by the compensatory growth of the skull and brain parallel to the premature fused skull suture. Patients with craniosynostosis syndromes often develop ventriculomegaly, which may induce periventricular WM atrophy but also changes the shape of surrounding brain structures. Particularly the corpus callosum is at risk for CSF contamination, and DTI FT has previously been described as being difficult to conduct in patients with hydrocephalus. ${ }^{28}$ Hence, difficulties in fiber tracking is (among others) caused by partial volume effects, we discovered that DTI FT in patients with craniosynostosis syndromes is more challenging than in controls; the combination of structural brain abnormalities and anisotropic voxels gives rise to less realistic fiber tract reconstructions. Unfortunately less is known about the effects of anisotropic voxels on the outcome of the FT, though it is assumed that isotropic voxels may be more beneficial for FT. ${ }^{29}$ The use of an anisotropic voxel size in our study might be seen as a limitation; however, FT in our control group without skull shape abnormalities did not cause any difficulties. Therefore, we believe that the deformity of WM structures itself caused by the genetic defect, prematurely fused skull sutures, and/or large CSF spaces nearby has a greater influence on fiber tract reconstructions than the anisotropic voxel size.

Another limitation of our study includes the inability for total blinding of the observers; the altered brain shape in our patient population could often be visually detected during the measurements. In addition, we were able to include only a small number of control subjects within the same age range as our patients with craniosynostosis syndromes, who were not age and sex matched. However, because the age of all subjects ranged from 6 to 18 years and the brain has matured thoroughly enough from 6 years of age to yield stable anisotropic indexes, DTI properties seem to change only slightly afterward.

Regarding DTI properties between controls and patients with craniosynostosis syndromes, we found that FA was similar between both groups, in contrast to the findings in the study of Florisson et al. ${ }^{7}$ Our study showed lower FA in almost all structures of both controls and patients with craniosynostosis syndromes, probably caused by the different postprocessing pipeline and data-acquisition protocol. In addition, placing a single ROI may be more subjective and could cause an overestimate of the FA of the structure of interest. Furthermore, the FA is typically underestimated in areas with crossing fibers and is further affected by an anisotropic voxel size. ${ }^{29,30}$ Comparable with results of the study of Florisson et al, ${ }^{7}$ diffusivity properties of the mean WM in our study were significantly higher in the total group of patients with craniosynostosis syndromes than in the control group. Although diffusivity parameters per craniosynostosis syndrome were higher than those in controls as well, regarding particular WM tracts there was no clear distinction between any craniosynostosis syndrome and the control group. One could argue that this is remarkable because FGFR genes have a major influence on myelinization of WM tracts by involving the development of oligodendrocytes, ${ }^{31}$ while the TWIST1 gene responsible for SaethreChotzen syndrome is important in mesenchymal cell lineage. ${ }^{32,33}$

Similar to the findings in the study of Yuan et $\mathrm{al}^{34}$ regarding DTI in infants with hydrocephalus, the frontal occipital horn ratio (ie, ventricular size) was significantly related to a lower FA and higher diffusivity properties in the genu and corpus of the corpus callosum in our study. This finding might be caused by the increased amount of water or edema in the extracellular space, ${ }^{35,36}$ because these structures are located closest to the ventricles. We assume that the role of the central CSF spaces is at least as big as the genetic influence in causing WM alterations. As in patients with sagittal craniosynostosis in whom altered DTI parameters may underlie their neuropsychological deficits, ${ }^{37} \mathrm{WM}$ abnormalities of the cingulate gyrus and corpus callosum in syndromic patients with craniosynostosis syndromes may be responsible for existing attention and memory problems. ${ }^{14}$ However, neurologic assessment of our patients with craniosynostosis syndromes cannot explain diffusivity abnormalities in the corticospinal tracts, and motor deficits might rather be caused by impair- 
ment of the frontal WM. ${ }^{38}$ Remarkably, the fornix seems to be spared by the mechanical pressure or stretching caused by ventriculomegaly and altered brain shape. By contrast, Hattori et $\mathrm{al}^{13}$ did show reduced FA values in the fornix of patients with normal pressure hydrocephalus compared with controls.

If we take these results together, our findings demonstrate that patients with craniosynostosis syndromes have a normal fiber organization but exhibit abnormal diffusivity values that may be related to differences in microstructural tissue properties. Performing DTI FT in very young patients with craniosynostosis syndromes without an operation, in whom secondary changes of the WM microarchitecture are unlikely to have occurred yet, would be interesting, to relate WM disturbances to either genetic influences or secondary changes including enlarged ventricles.

\section{CONCLUSIONS}

DTI FT is challenging to perform in patients with craniosynostosis syndromes, most likely because of their deformed brain and abnormal ventricular size and shape. This study showed that patients with craniosynostosis syndromes have FA equal to that in control subjects, while $\mathrm{MD}, \mathrm{AD}$, and $\mathrm{RD}$ were significantly higher in different brain structures in these patients. Although these differences may indicate abnormalities in tissue microstructural properties, such as myelin deficiency and axonal loss, we cannot exclude confounding contributions of partial volume effects related to the enlarged CSF spaces. No craniosynostosis syndromespecific differences in DTI properties were seen in any particular type of fiber tract.

\section{REFERENCES}

1. Johnson D, Wilkie AO. Craniosynostosis. Eur J Hum Genet 2011;19:369-76

2. Di Rocco C, Frassanito P, Massimi L, et al. Hydrocephalus and Chiari type I malformation. Childs Nerv Syst 2011;27:1653-64

3. Britto JA, Evans RD, Hayward RD, et al. From genotype to phenotype: the differential expression of FGF, FGFR, and TGFbeta genes characterizes human cranioskeletal development and reflects clinical presentation in FGFR syndromes. Plast Reconstr Surg 2001; 108:2026-39; discussion 2040-46

4. Raybaud C, Di Rocco C. Brain malformation in syndromic craniosynostoses, a primary disorder of white matter: a review. Childs Nerv Syst 2007;23:1379-88

5. de Jong T, Rijken BF, Lequin $\mathrm{MH}$, et al. Brain and ventricular volume in patients with syndromic and complex craniosynostosis. Childs Nerv Syst 2012;28:137-40

6. Morriss-Kay GM, Wilkie AO. Growth of the normal skull vault and its alteration in craniosynostosis: insights from human genetics and experimental studies. J Anat 2005;207:637-53

7. Florisson JM, Dudink J, Koning IV, et al. Assessment of white matter microstructural integrity in children with syndromic craniosynostosis: a diffusion-tensor imaging study. Radiology 2011;261: $534-41$

8. Cinalli G, Renier D, Sebag G, et al. Chronic tonsillar herniation in Crouzon's and Apert's syndromes: the role of premature synostosis of the lambdoid suture. J Neurosurg 1995;83:575-82

9. Cinalli G, Spennato P, Sainte-Rose C, et al. Chiari malformation in craniosynostosis. Childs Nerv Syst 2005;21:889-901

10. Jeevan DS, Anlsow P, Jayamohan J. Abnormal venous drainage in syndromic craniosynostosis and the role of CT venography. Childs Nerv Syst 2008;24:1413-20
11. Quintero-Rivera F, Robson CD, Reiss RE, et al. Intracranial anomalies detected by imaging studies in $\mathbf{3 0}$ patients with Apert syndrome. Am J Med Genet A 2006;140:1337-38

12. Cohen MM Jr. Pfeiffer syndrome update, clinical subtypes, and guidelines for differential diagnosis. Am J Med Genet 1993;45: 300-07

13. Hattori $\mathrm{T}$, Ito $\mathrm{K}$, Aoki $\mathrm{S}$, et al. White matter alteration in idiopathic normal pressure hydrocephalus: tract-based spatial statistics study. AJNR Am J Neuroradiol 2012;33:97-103

14. Maliepaard M, Mathijssen IM, Oosterlaan J, et al. Intellectual, behavioral, and emotional functioning in children with syndromic craniosynostosis. Pediatrics 2014;133:e1608-15

15. Basser PJ, Mattiello J, LeBihan D. MR diffusion tensor spectroscopy and imaging. Biophys J 1994;66:259-67

16. Beaulieu $C$. The basis of anisotropic water diffusion in the nervous system: a technical review. NMR Biomed 2002;15:435-55

17. Le Bihan D. Looking into the functional architecture of the brain with diffusion MRI. Nat Rev Neurosci 2003;4:469-80

18. van Engelen SJ, Krab LC, Moll HA, et al. Quantitative differentiation between healthy and disordered brain matter in patients with neurofibromatosis type I using diffusion tensor imaging. AJNR Am J Neuroradiol 2008;29:816-22

19. Leemans A, Jeurrissen B, Sijbers J, Jones DK. ExploreDTI: a graphical toolbox for processing, analyzing, and visualizing diffusion MR data. In: Proceedings of the 17th Annual Meeting of International Society of Magnetic Resonance in Medicine, Honolulu, Hawaii. April 1824, 2009:3537

20. Leemans A, Jones DK. The B-matrix must be rotated when correcting for subject motion in DTI data. Magn Reson Med 2009;61: $1336-49$

21. Tax CM, Otte WM, Viergever MA, et al. REKINDLE: robust extraction of kurtosis INDices with linear estimation. Magn Reson Med 2015;73:794-808

22. Veraart J, Sijbers J, Sunaert S, et al. Weighted linear least squares estimation of diffusion MRI parameters: strengths, limitations, and pitfalls. Neuroimage 2013;81:335-46

23. Oishi K, Faria AV, van Zijl PC, et al. MRI Atlas of Human White Matter. 2nd ed. Amsterdam: Elsevier; 2010

24. Basser PJ, Pajevic S, Pierpaoli C, et al. In vivo fiber tractography using DT-MRI data. Magn Reson Med 2000;44:625-32

25. Malykhin N, Concha L, Seres P, et al. Diffusion tensor imaging tractography and reliability analysis for limbic and paralimbic white matter tracts. Psychiatry Res 2008;164:132-42

26. Kumar A, Sundaram SK, Sivaswamy L, et al. Alterations in frontal lobe tracts and corpus callosum in young children with autism spectrum disorder. Cereb Cortex 2010;20:2103-13

27. Reijmer YD, Leemans A, Heringa SM, et al. Improved sensitivity to cerebral white matter abnormalities in Alzheimer's disease with spherical deconvolution based tractography. PLoS One 2012;7:e44074

28. Rajagopal A, Shimony JS, McKinstry RC, et al. White matter microstructural abnormality in children with hydrocephalus detected by probabilistic diffusion tractography. AJNR Am J Neuroradiol 2013;34:2379-85

29. Oouchi H, Yamada K, Sakai K, et al. Diffusion anisotropy measurement of brain white matter is affected by voxel size: underestimation occurs in areas with crossing fibers. AJNR Am J Neuroradiol 2007;28:1102-06

30. Vos SB, Jones DK, Jeurissen B, et al. The influence of complex white matter architecture on the mean diffusivity in diffusion tensor MRI of the human brain. Neuroimage 2012;59:2208-16

31. Furusho M, Dupree JL, Nave KA, et al. Fibroblast growth factor receptor signaling in oligodendrocytes regulates myelin sheath thickness. J Neurosci 2012;32:6631-41

32. Chen $\mathrm{ZF}$, Behringer RR. Twist is required in head mesenchyme for cranial neural tube morphogenesis. Genes Dev 1995;9:686-99

33. el Ghouzzi V, Le Merrer M, Perrin-Schmitt F, et al. Mutations of the 
TWIST gene in the Saethre-Chotzen syndrome. Nat Genet 1997; 15:42-46

34. Yuan W, Mangano FT, Air EL, et al. Anisotropic diffusion properties in infants with hydrocephalus: a diffusion tensor imaging study. AJNR Am J Neuroradiol 2009;30:1792-98

35. Berlot R, Metzler-Baddeley C, Jones DK, et al. CSF contamination contributes to apparent microstructural alterations in mild cognitive impairment. Neuroimage 2014;92:27-35
36. Vos SB, Jones DK, Viergever MA, et al. Partial volume effect as a hidden covariate in DTI analyses. Neuroimage 2011;55:1566-76

37. Beckett JS, Brooks ED, Lacadie C, et al. Altered brain connectivity in sagittal craniosynostosis. J Neurosurg Pediatr 2014;13:690-98

38. Lenfeldt N, Larsson A, Nyberg L, et al. Diffusion tensor imaging reveals supplementary lesions to frontal white matter in idiopathic normal pressure hydrocephalus. Neurosurgery 2011;68:1586-93; discussion 1593 